GRUPO DE TRABAJO RIIER*

Red Internacional de Investigadores sobre Encerramientos Residenciales (Cali, Colombia)

\title{
Red Internacional de Investigadores sobre Encerramientos Residenciales (RIIER)
}

* E-mail: redencerramientoresidencial@gmail.com |Web: http://redinvestigacionerm.blogspot.com/ 
En las últimas décadas la urbanización en el mundo ha experimentado grandes cambios demográficos, económicos, tecnológicos y sociales, generando auténticas transformaciones territoriales tanto de tipo urbanístico como social. Procesos socio-espaciales en territorios metropolitanos de todo el planeta, con patrones de segregación, fragmentación y policentrismo que constituyen auténticos «archipiélagos metropolitanos» y a escala planetaria configurarían una «constelación urbana» son identificados por relevantes autores e investigaciones, en asociación a las tipologías urbanísticas y arquitectónicas del condominio residencial cerrado como artefacto y dispositivo predilecto del capitalismo contemporáneo y de la globalización.

Se consideran los impactos crecientes en todo el mundo de los denominados «barrios cerrados», «ciudades valladas», «condominios cerrados», «encerramientos residenciales» $\mathrm{O}$ «fraccionamientos», y su correlato en las nuevas relaciones sociales internas de estos conjuntos, como con las relaciones con el vecindario y con las nuevas estructuras metropolitanas. La consolidación de los «encerramientos residenciales» no sólo estaría afectando los modos de convivencia, sino que también estaría ocasionando una nueva re-conceptualización de la noción de barrio e impactando la estructura, forma y funcionalidades del territorio.

La coincidencia de intereses en esta temática de varios docentes de universidades de la ciudad de Santiago de Cali motivó la celebración, los días 13 y 14 de octubre de 2015, del I Seminario Internacional sobre Encerramiento Residencial, evento organizado por la Escuela de Trabajo Social de la Universidad del Valle que contó con la participación de dos sobresalientes invitadas internacionales: las profesoras Sonia Roitman, de la Universidad del Quensland (Australia), y de Edvania Torres, de la Universidad Federal de Pernambuco (Brasil); también de un grupo de docentes e investigadores del país, de actores y agentes locales de los sectores privado y público relacionados con distintas temáticas asociadas a los condominios residenciales cerrados en la ciudad de Cali, y de un auditorio que representaba intereses tanto de la académia como de la sociedad civil.

El Seminario fue estructurado en cuatro ejes temáticos de especial relevancia para la comprensión holística del objeto abordado: el primero acerca de los impactos en la estructura y la morfología del territorio metropolitano; el segundo referido a su conexión con la inseguridad objetiva y subjetiva que se vive en el contexto urbano latinoamericano; el tercero ligado a su ensamble con el espacio público de proximidad; y el cuarto relacionado con la convivencia al interior de estos espacios habitacionales. Estos ejes temáticos se desarrollaron en cuatro mesas de trabajo que recogieron las ponencias enviadas: Mesa 1: Conjuntos Residenciales Encerrados: morfología y funciones urbanas de la ciudad contemporánea-ciudad fragmentada-; Mesa 2: Encerramientos Residenciales y seguridad; Mesa 3: Encerramiento Residencial y Espacio Público; Mesa 4: Convivencia y gobernabilidad en encerramientos residenciales. Las ponencias fueron reconstruidas como artículos científicos y publicadas en la Revista Prospectiva número 21 de abril de 
2016, ${ }^{1}$ monográfico sobre el tema del encerramiento residencial con página editorial de la doctora Sonia Roitman.

En el marco del primer seminario sobre el tema, se formalizó la constitución de una red de Investigación internacional con el ánimo de promover un escenario para la reflexión interdisciplinar e interinstitucional sobre el fenómeno habitacional. La Red Internacional de Investigación sobre Encerramientos Residenciales (RIIER) ${ }^{2}$ se planteó el propósito de estrechar vínculos entre investigadores e instituciones académicas de todo el mundo para promover la reflexión, el debate, la producción e intercambio de conocimiento a través de publicaciones, encuentros, seminarios, foros alrededor de la temática de los encerramientos residenciales urbanos.

La RIIER ${ }^{3}$ invita a todas aquellas instituciones, grupos de investigación, investigadores y personas interesadas en el estudio y las problemáticas de los encerramientos residenciales a sumarse a esta iniciativa académica.

El segundo seminario internacional se realizará en la ciudad de Medellín (Colombia) los días 11 y 12 de octubre de 2018. Se espera una amplia participación de conferencistas y ponentes investigadores de diferentes países como también representantes del sector público y privado (gremios de la construcción), y de la sociedad civil, para reflexionar

1. Revista de Trabajo Social e Intervención Social de la Escuela de Trabajo Social de la Facultad de Humanidades de la Universidad del Valle, $\mathrm{N}^{\circ}$ 21, se puede consultar en https://goo.gl/ReH3Iq

2. Los participantes de la protocolización de la Red Encerramiento Residencial son: Edvania Torres Aguiar Gomes, profesora de la Universidad Federal de Pernambuco (Brasil). E-mail: torres@ufpe.br; Sonia Roitman, profesora de la The University of Queensland (Australia).E-mail: s.roitman@uq.edu.au; María Teresa Rincón, profesora de la Escuela de Trabajo social de la Universidad del Valle (Colombia). E-mail: maria.rincon@ correounivalle.edu.co; Martha Lucía Echeverry, profesora de la Escuela de Trabajo social de la Universidad del Valle (Colombia). E-mail: marluev660@gmail.com; Adolfo García, profesor de la Facultad de Ciencias Sociales y Económicas de la Universidad del Valle (Colombia). E-mail: adolfo.garcia@correounivalle.edu. co; Carlos Botero Restrepo, profesor de la Escuela de Arquitectura de la Universidad del Valle (Colombia). E-mail: carlos.botero@correounivalle.edu.co; María Cristina Maldonado, trabajadora social de la Universidad del Valle (Colombia). E-mail: maria.cristina.maldonado@correounivalle.edu.co; Hermes Martínez Barrios, profesor de la Universidad Popular del Cesar (Colombia).E-mail: hermesmartinez19@yahoo.com; Hernando Uribe Castro, profesor de la Universidad Autónoma de Occidente (Colombia). E-mail: huribe@uao.edu.co; Enrique Rodríguez Caporali, profesor de la Universidad Icesi (Colombia).E-mail: caporali@icesi.edu.co; Juan Manuel Castañeda Vega Arquitecto-urbanista, Universidad Nacional de Colombia E-mail: jumaca81@gmail. com; Pedro Martínez Toro, profesor del Departamento de Geografía de la Universidad del Valle (Colombia). E-mail:pedro.martinez@correounivalle.edu.co; Sabina Cárdenas O’byrne, profesora de la Facultad de Arquitectura de la Pontificia Universidad Javeriana (Colombia).E-mail sabinac@javerianacali.edu.co; Oscar Becerra Sociólogo, arquitecto de la Universidad del Valle (Colombia).E-mail: arcillaecoacabados@gmail.com; Aura González, profesora de la Universidad Pontificia Bolivariana (Colombia).E-mail: aura.gonzalez@upb.edu.co.

3. Mayor información sobre la Red en el E-mail: redencerramientoresidencial@gmail.com o en el Blog: http:// redinvestigacionerm.blogspot.com/ 
y discutir desde diferentes perspectivas sobre las distintas aristas del fenómeno y en particular sobre el impacto económico, político, social, cultural y ambiental de esta forma de habitar la ciudad.

\section{IMAGEN 1 | Registro del acto de constitución de la RIIER - Cali, agosto 14 de 2015}

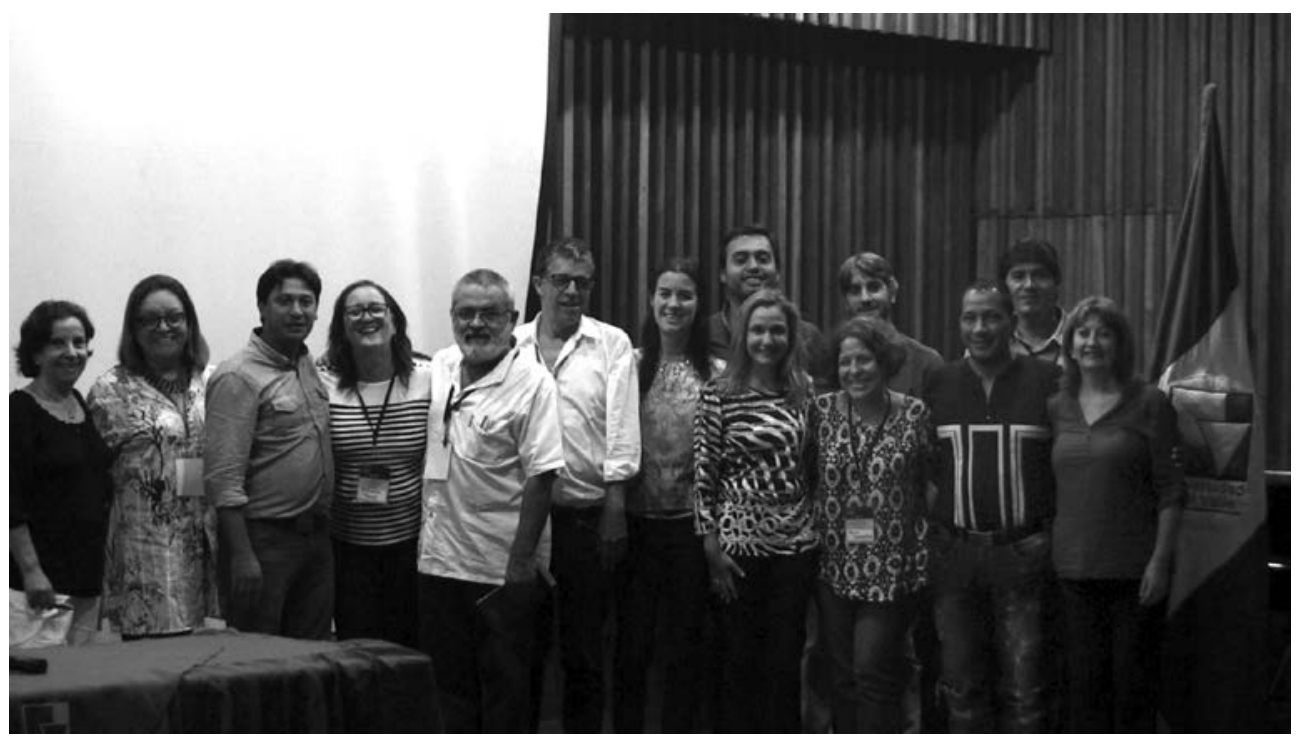

Fuente: tomada por el grupo de trabajo de la RIIER. 